\title{
Un derecho ambiental democrático para Latinoamérica y el Caribe: los retos de la negociación del Acuerdo Regional sobre el Principio 10 de Río 92
}

Fecha de recepción: 28 de febrero de 2017

Fecha de aceptación: 11 de diciembre de 2017

DOI: dx.doi.org/10.12804/revistas.urosario.edu.co/acdi/a.6541

\author{
Erika Castro-Buitrago* \\ Felipe Calderón Valencia ${ }^{* *}$
}

Resumen: el objetivo de este artículo es contribuir con el relato analítico-crítico de la construcción del derecho ambiental democrático latinoamericano y del Caribe, abordándolo como construcción histórica en dos etapas que parten de Río 92 (planeación) y de Río+20 (concreción). Se cuestiona si la región superó la planeación y pasó a la concreción de estándares para garantizar el principio 10 con la firma de un acuerdo regional. Para alcanzar el objetivo, se sigue una investigación dogmática de corte descriptivo, analítico y prescriptivo, usando la técnica de análisis documental, privilegiando fuentes primarias y la observación de las negociaciones del acuerdo

* Doctora en Medio Ambiente y Ordenación del Territorio de la Universidad Autónoma de Madrid. Profesora de la Universidad de Medellín, Medellín (Colombia). Correo electrónico: ecastro@udem.edu.co. http://orcid.org/0000-0002-0467-0031.

** Doctor en Derecho de la Universidad Panthéon-Assas (París II). Profesor de la Universidad de Medellín, Medellín (Colombia). Correo electrónico: fcalderon@udem.edu.co. http://orcid.org/0000-0001-7384-7470.

Para citar este artículo: Castro-Buitrago, E. \& Calderón Valencia, F., "Un derecho ambiental democrático para Latinoamérica y el Caribe: los retos de la negociación del Acuerdo Regional sobre el Principio 10 de Río 92", ACDI-Anuario Colombiano de Derecho Internacional, 2018, 11, pp. 159-186.

DOI: dx.doi.org/10.12804/revistas.urosario.edu.co/acdi/a.6541 
regional. Esto es resultado de una investigación que busca caracterizar la justicia ambiental y su acceso en Latinoamérica.

Palabras clave: principio 10, derechos de acceso, acuerdo regional, democracia ambiental.

\section{A Democratic Environmental Law for Latin America and the Caribbean: The Challenges of Negotiating the Regional Treaty of the Principle 10 of Rio 92}

Abstract: The objective of this article is to contribute with the analyticalcritical construction of democratic environmental law in Latin America, addressing it as a historical construction in two different stages, starting with Rio 92 (planning) and Rio +20 (concretion). The main question is to find out if this region already exceeded planning and went into a concretion stage by guaranteeing standards of Principle 10 by signing a regional agreement. To achieve this objective, a dogmatic research of a descriptive, analytical and prescriptive nature is followed, using the technique of primary documentary sources analysis and observing the negotiations of the regional agreement. This is the result of a research characterizing environmental justice and the issue of the access to it in Latin America.

Keywords: Principle 10, access rights, regional agreement, environmental democracy.

Um direito ambiental democrático para a América Latina e o Caribe: os desafios da negociação do Acordo Regional sobre o Princípio 10 do Rio 92

Resumo: o objetivo deste artigo é contribuir com o relato analítico-crítico da construção do direito ambiental democrático latino-americano e do Caribe, abordando-o como construção histórica em duas etapas que partem do Rio 92 (planejamento) e do Rio+20 (concreção). Questiona-se se a região superou o planejamento e passou à concreção de standards para garantir o Princípio 10 com a assinatura do acordo regional. Para alcançar o objetivo se segue uma pesquisa dogmática de corte descritivo, analítico e prescritivo, usando a técnica de análise documental, privilegiando fontes 
primárias e a observação das negociações do acordo regional. Isto é resultado de uma pesquisa que busca caracterizar a justiça ambiental e o seu acesso na América Latina.

Palavras-chave: princípio 10, direitos de acesso, acordo regional, democracia ambiental.

\section{Introducción}

El propósito del presente artículo es contribuir con el relato analítico-crítico de la construcción de un derecho ambiental democrático para Latinoamérica y el Caribe. Para realizar este trabajo, se siguió una investigación dogmática de corte descriptivo, analítico y prescriptivo. La metodología propuesta parte de la descripción de los textos jurídicos base de la negociación del Acuerdo Regional sobre el Principio $10^{1}$ (en adelante, ARP10) de la Declaración de Río de Janeiro del 14 de junio de $1992^{2}$ (en adelante, Río 92), la observación directa de la negociación y los documentos producidos en su desarrollo (fase descriptiva) con base en la técnica de análisis documental; el contraste de este material con los conceptos jurídicos y estándares internacionales en la materia (fase analítica); para, finalmente, hacer su valoración crítica y propositiva (fase prescriptiva). ${ }^{3}$

Para alcanzar este objetivo, se proponen dos etapas: una de tipo descriptivo-crítico y otra analítico-propositiva. Las etapas propuestas buscan, mediante el tratamiento privilegiado de fuentes primarias, valorar los retos de la negociación del ARP10, cuya etapa final se espera que llegue con posterioridad a la Octava Reunión del Comité de Negociación del Acuerdo Regional sobre el Acceso a la Información, la Participación

\footnotetext{
1 Organización de las Naciones Unidas, "Declaración de Río sobre el Medio Ambiente y el Desarrollo", 14 de junio de 1992, en http://www.un.org/spanish/esa/sustdev/documents/declaracionrio.htm, consulta del 25 de febrero de 2017.

2 Cabrera, Jorge, "El impacto de las declaraciones de Río y Estocolmo sobre la legislación y las políticas ambientales en América Latina", Revista Interdisciplinar de Gestión Ambiental, 2004, 6, (61).

3 Resultado del proyecto de investigación: "Caracterización de la justicia formal ambiental y del derecho de acceso a la justicia: un estudio comparado de los ordenamientos jurídicos latinoamericanos para proponer una revisión de la justicia formal ambiental en Colombia”. Financiado por la Universidad de Medellín (2016-2018).
} 
Pública y el Acceso a la Justicia en Asuntos Ambientales en América Latina y el Caribe (27 de noviembre al $1^{\circ}$ de diciembre de 2017).

Una de las causas de la actual crisis ambiental es el modelo de desarrollo global y la manera tradicional de abordar los asuntos ambientales desde la polarización Estado y sociedad. ${ }^{4}$ Estas han sido materia de reflexión en las llamadas Cumbres de la Tierra (en adelante, Cumbres) que inspiran el derecho ambiental democrático puesto a consideración de los Estados del continente americano desde hace cinco años y gracias a Río 92. Su filosofía se funda en el reconocimiento y respeto de la diferencia, estableciendo normas para involucrar a todos los actores interesados en la construcción de un futuro mejor.

Tal paradigma tiene dos etapas de formación que serán explicadas a continuación, donde la negociación del ARP10 marca un antes y un después. Pasadas ocho reuniones de dicha negociación, cabe preguntarse: ¿Los Estados de Latinoamérica y el Caribe están realmente en una etapa de concreción de lo planeado en las Cumbres? Esta primera etapa busca la protección efectiva de los llamados derechos de acceso (acceso a la información, participación y justicia en asuntos ambientales) que tienen por fin "reducir las brechas de implementación del principio 10". 5

Para dar una explicación sistemática de esta historia del derecho internacional ambiental, se plantea una estructura bipartita. La primera parte brinda una visión general de dos importantes cumbres organizadas por la ONU, cuyo primer resultado fue Río 92 y el segundo, la Declaración de Río de $2012^{6}$ (en adelante Río+20). La segunda parte de este artículo explica los desafíos de la negociación de un instrumento que busca establecer estándares mínimos de protección de los derechos de acceso en la región. Esto se logra con el análisis de la negociación y las intervenciones de los actores, en contraste con los documentos de preparación y los diferentes instrumentos de derechos humanos relacionados con la protección ambiental.

\footnotetext{
4 Ost, Francoise, Naturaleza y derecho: para un debate ecológico en profundidad, Editorial Mensajero, Bilbao, 1996.

Comisión Económica para América Latina y el Caribe, Visión de Lima para un instrumento regional sobre los derechos de acceso en materia ambiental, NU. Cepal Doc. LC/L.3780, 21 de febrero de 2014, p. 2.

6 Organización de las Naciones Unidas, El futuro que queremos, NU. Doc. A/CONF.216/L.1, 19 de junio de 2012.
} 


\section{Construcción de un derecho ambiental para Latinoamérica y el Caribe}

Si la vida es el sustento de los demás derechos y esta es un derecho fundamental, resulta difícil no establecer una relación lógica entre el ambiente como sustento de la vida y el derecho ambiental como instrumento de su protección. En la transición del siglo XX al XXI, esta rama del derecho ha evolucionado para tratar de cerrar la brecha entre las comunidades, su ambiente y el Estado. Dicho ideal se impulsa con el precitado principio 10, que propone la democracia ambiental para dejar atrás el paradigma puramente técnico del derecho ambiental, en un contexto de evoluciones del orden constitucional interno fundado en la armonía con el orden jurídico internacional. ${ }^{7}$

Aunado a lo anterior, los retos impuestos por el cambio climático son otro factor que precipita mudar el paradigma técnico del derecho ambiental en Latinoamérica. ${ }^{8}$ No obstante, hablar de cambio abrupto y radical resulta problemático, porque desde Río 92 se ha dado una lenta evolución que puede explicarse en dos etapas.

La primera (1992-2010) es un momento de promesas y planificación (1.1), mientras que la segunda (desde 2012 hasta hoy) se caracteriza por la acción o la búsqueda en la adopción de un instrumento regional para la aplicación de los derechos de acceso derivados del principio 10 (1.2). ¿En cuál etapa se encuentran los Estados de Latinoamérica? Responder esta pregunta requiere plantear el debate sobre si los cambios introducidos desde Río 92 son estructurales o coyunturales.

\subsection{Etapa de planeación (1992-2010)}

Para entender esta etapa, hay que referenciar las Cumbres, fundamentalmente tres: la primera se llevó a cabo en Estocolmo en $1972{ }^{9}{ }^{\circ}$ en la

De Wet, Erika, “The international constitutional order", International and Comparative Law Quarterly, 2006, 55, (1), pp. 51-76.

8 Rosso, Margaret, "Climate change and the law", The American Journal of Comparative Law, 2010, 58, pp. 223-255.

9 Organización de las Naciones Unidas, "Declaration of the United Nations Conference on the Human Environment”, 16 de junio de 1972, en http:/ / www.unep.org/Documents. Multilingual/Default.asp?DocumentID=97\&ArticleID=1503\&l=en, consulta del 27 de febrero de 2017. 
segunda nace la Declaración de Río, en 1992; y la tercera, en 2002, en Johannesburgo. ${ }^{10}$ Estas son el marco histórico de un desarrollo del derecho ambiental que, paulatinamente, intenta cerrar la brecha entre ciudadanos, ambiente y los Estados.

Río 92 es un hito en razón de la exaltación de continuidad de lo pactado en Estocolmo y de la declaración de una serie de principios. Estos últimos fijaron nuevos parámetros de evolución del derecho ambiental interno a título de soft law ${ }^{11}$ (e. g., el Programa 21 y la Declaración de Río sobre el Medio Ambiente y el Desarrollo). Para los países de América Latina y el Caribe, aquellos fueron de vital importancia para configurar, hasta 2012, compromisos para un futuro que recién se concreta con la negociación del ARP10.

Ciertamente, los Estados reunidos en Brasil sentaron las bases de un marco legal de la ecopolítica que liga medio ambiente y desarrollo, ${ }^{12}$ pero si bien los ambiciosos propósitos de Río 92 no han adoptado una forma jurídica vinculante, la segunda cumbre ejerció cierta presión sobre Latinoamérica y el Caribe para trabajar en pos de un modelo de desarrollo alterno. ${ }^{13}$ Los 27 principios de Río 92 reafirman lo dicho en Estocolmo y se transformaron en el ariete jurídico de una crítica al desarrollo desligado de la sostenibilidad. ${ }^{14}$

Para agregar un par de ejemplos de Río 92, el principio 1 fundamenta jurídicamente la ecopolítica, empleando el concepto de 'vida saludable' y no únicamente el de vida; y el principio 2 fija —apoyado en el derecho internacional y la Carta de la ONU — una responsabilidad de cuidado del ambiente, ligada al ejercicio tradicional de la soberanía. ${ }^{15}$ Así, en primer

10 Organización de las Naciones Unidas, Plan de aplicación de las decisiones de la Cumbre Mundial sobre el Desarrollo Sostenible Johannesburgo (Sudáfrica), 26 de agosto al 4 de septiembre de 2002, Nu. Doc. A/CONF.199/L.3/Add.1-13, 4 de septiembre de 2002.

11 Lozano, Blanca, Derecho ambiental administrativo, Dykinson, Madrid, 2007, p. 53.

12 Comisión Económica para América Latina y el Caribe, Río 92 y la realidad latinoamericana. Una mirada a la crisis ambiental desde el sur, NU. Cepal Doc. LC/R.1140, 4 de mayo de 2002, p. 3; Bryant, Raymond; "Beyond the impasse: the power of political ecology in Third World environmental research", Area, 1997, 29, (1), pp. 5-19.

13 Organización de las Naciones Unidas, "Declaración de Río sobre el Medio Ambiente...", op. cit.

14 Organización de las Naciones Unidas, El futuro que queremos, op. cit.

15 Sands, Philipe, Peel, Jacqueline, Fabra, Adriana \& MacKenzie, Ruth, Principles of international environmental law, Cambridge University Press, Nueva York, 2012, p. 190. 
lugar, el discurso subyacente del período 1992-2010 tempera el discurso clásico del desarrollo sin sostenibilidad, inherente a la etapa de planeación. En segundo lugar, el compromiso de erradicar la pobreza, propuesta de Río 92, se propone desde un esquema de responsabilidad con el ambiente que promueva nuevas formas de consumo cada vez menos agresivas. ${ }^{16}$

Entonces, los cimientos del período 1992-2010 están puestos en principios promovidos en el interior de los Estados de la región pese a que no han sido plenamente adoptados en los ordenamientos jurídicos internos. ${ }^{17} \mathrm{El}$ soft law de Río 92 condiciona el devenir del nuevo derecho ambiental y su finalidad es promover la ecopolítica e intereses de un modelo de desarrollo alternativo.

Se agrega que varios eventos tributarios de esta lógica tuvieron lugar a finales del siglo Xx. El primero, derivado de Río 92, fue la Cumbre para la Tierra +5 de 1997, ${ }^{18}$ donde la Asamblea General de la ONU aprueba el Programa $21 .{ }^{19}$ Igualmente, de la reunión se derivó la agenda del período 1998-2002 de la llamada Comisión sobre el Desarrollo Sostenible, ${ }^{20}$ definiendo lo que sería la Tercera Cumbre.

En consecuencia, otro evento prepara el terreno para el desarrollo del nuevo derecho ambiental a comienzos del siglo xxi: la Cumbre Mundial sobre el Desarrollo Sostenible de Johannesburgo. Sus reuniones finales dejaron importantes documentos para impactar las políticas de los Estados y, así, pasar de la planificación a la acción. Dentro de esta lista de declaraciones, el llamado Plan de Aplicación de las Decisiones de Johannesburgo ${ }^{21}$ propone medidas que parecen más concretas referenciando abundantemente otros instrumentos de la ONU. Allende fijar metas, se

16 Organización de las Naciones Unidas, El futuro que queremos, op. cit., p. 1; Porter, John, Howden, Mark, Smith, Pete \& Stokholm, Michaela, "Recasting economics as if the climate and global ecology really mattered”, Consilience, 2017, (17), pp. 220-229.

17 Cabrera, "El impacto de las declaraciones...", op. cit., pp. 30-38.

18 Organización de las Naciones Unidas, "Cumbre para la Tierra +5, período extraordinario de sesiones de la Asamblea General para el examen y la evaluación de la aplicación del Programa 21", Nueva York, 23 a 27 de junio de 1997, en http:/ /www.un.org/spanish/ conferences/cumbre\&5.htm, consulta del 25 de febrero de 2017.

19 Organización de las Naciones Unidas, "Programa 21", en http:/ / www.un.org/spanish/ esa/sustdev/agenda21/, consulta del 25 de febrero de 2017.

20 Organización de las Naciones Unidas, "Cumbre para la Tierra $+5 \ldots$... op. cit.

21 Organización de las Naciones Unidas, Plan de aplicación de las decisiones de la Cumbre Mundial sobre el Desarrollo Sostenible Johannesburgo..., op. cit. 
trazan objetivos cuantificables y ciertos plazos para la realización efectiva de Río $92 .{ }^{22}$

Recuérdese que el período 1992-2010 es de planeación, porque buscaba confrontar a la comunidad internacional con problemas ambientales reales. En efecto, el informe de la Tercera Cumbre refleja una insatisfacción frente al avance de lo pactado en las diferentes declaraciones. ${ }^{23}$

En suma, Johannesburgo es el balance de la primera etapa del desarrollo del derecho ambiental regional. Muestra que el período 1992-2010 es de promesas, sin un compromiso de fondo de los Estados.

\subsection{Etapa de concreción (2012-2017)}

¿Qué quedó luego de Río 92 en el siglo Xxi? La respuesta es Río+20, a pesar de la crisis de la gobernanza, ${ }^{24}$ tal y como se observa desde las críticas a la inoperancia de ciertos Estados frente al nuevo modelo de desarrollo. ${ }^{25}$ Diez años después de la Tercera Cumbre, los intentos por fijar los alcances de un derecho ambiental más democrático en Latinoamérica todavía provienen del ámbito internacional. Río +20 impulsó la producción de un derecho interno que atendiera los fines comunes de un desarrollo amigable con el ambiente, pero también propició la Declaración sobre la Aplicación del Principio $10^{26}$ (en adelante DP10). Con esta nueva declaración los Estados

22 Bastida, Ana \& Bustos, Luis, "Towards regimes for sustainable mineral resource management. Constitutional reform, law and judicial decisions in Latin America", en Carbonnier, G., Campodónico, H. \& Vázquez, S. T. (eds.), Alternative pathways to sustainable development: lessons from Latin America, Brill, Geneva, 2017, pp. 242-244.

23 Godard, Oliver, "Le développement durable de Río de Janeiro (1992) à Johannesburg (2002)", CECO-803, 2003, p. 5, en https://halshs.archives-ouvertes.fr/hal-00242971/document, consulta del 25 de febrero de 2017.

24 Guzmán, Patricia, Tratados ambientales internacionales y negocios, Universidad Externado de Colombia, Bogotá, 2012, p. 54; Castro Buitrago, Erika, Derecho ambiental y gobernanza. La concertación como actuación informal de la Administración, Universidad de Medellín, Medellín, 2017, pp. 154-161.

25 Godard, "Le développement...", op. cit., pp. 5-6.

26 Organización de las Naciones Unidas, Declaración sobre el Principio 10: Acceso a la Información, Participación y Justicia en Asuntos Ambientales en América Latina y el Caribe, NU. Doc. A/ CONF.216/13, 25 de julio de 2012. 
se comprometieron a dar inicio al proceso de creación del ARP10, para que fuera abierto, transparente y con inclusión del público latinoamericano. ${ }^{27}$

Comparándolos con los anteriores, los compromisos de la segunda etapa son más serios por dos razones. Primera: la acción positiva conlleva la discusión de un instrumento para aplicar los derechos de acceso. Segunda: la adopción de una serie de nuevos documentos fundamentales: la Hoja de Ruta (2012), el Plan de Acción (2013), la Visión de Lima (2012), las Líneas de Acción Prioritarias en Materia de Fortalecimiento de Capacidades y Cooperación (2013), los Contenidos de San José (2014) y la Decisión de Santiago (2014).

Por su parte, la Hoja de Ruta y las Líneas de Acción tenían por finalidad trazar un itinerario común ${ }^{28}$ (apoyado por la Cepal) ${ }^{29}$ y la identificación de buenas prácticas de los derechos de acceso en la región. Sumados los esfuerzos de Río +20 , puede entenderse fácilmente que la segunda etapa propicia el actuar condicionado a compromisos de la primera. La sistematicidad propia de los documentos emanados de Río+20 se percibe en los principios de los Contenidos de San José; citan la Visión de Lima para afirmar que la igualdad, la inclusión y la transparencia deben guiar la confección del instrumento regional. ${ }^{30}$

Una serie de directrices fueron aprobadas desde 2012 para crear el ARP10. ${ }^{31}$ En efecto, los instrumentos precitados fijaban un estándar

27 "[...] cualquier persona natural o jurídica u organizada en formas comunitarias" que posee el derecho de "participar en las reuniones [...]". Comisión Económica para América Latina y el Caribe, Plan de acción hasta 2014 para la implementación de la Declaración sobre la Aplicación del Principio 10 de la Declaración de Río sobre el Medio Ambiente y el Desarrollo en América Latina y el Caribe y su hoja de ruta, Nu. Cepal Doc., 17 de abril de 2013, p. 12.

28 Ibid., p. 4.

29 Conforme la Resolución 648 (XXXIII) de la Cepal, esta actuará como secretaría técnica de la negociación del ARP10, ibid., p. 11.

30 Comisión Económica para América Latina y el Caribe, Decisión de Santiago. Cuarta reunión de los puntos focales designados por los gobiernos de los países signatarios de la Declaración sobre la Aplicación del Principio 10 de la Declaración de Río sobre el Medio Ambiente y el Desarrollo en América Latina y el Caribe, Nu. Cepal Doc., 7 de noviembre de 2014.

Comisión Económica para América Latina y el Caribe, Informe de la cuarta reunión de los puntos focales designados por los gobiernos de los paises signatarios de la Declaración sobre la Aplicación del Principio 10 de la Declaración de Río sobre el Medio Ambiente y el Desarrollo en América Latina y el Caribe, Nu. Cepal Doc. LC/L.3970, 12 de marzo de 2015, p. 24.

31 Conviene citar la adopción del Convenio de Aarhus (ratificado por la Comunidad Europea mediante la decisión del consejo del 17 de febrero de 2005) como un importante 
mínimo para la negociación. Comenzando por la Declaración sobre el Principio 10, Río+20 dejó claro que "[...] reconocemos que debemos alcanzar compromisos para la implementación cabal de los derechos de acceso". ${ }^{32}$ El mensaje de las partes conjura la poca eficacia de Río 92, invita a pasar del soft law al hard law.

Por su parte, la Visión de Lima fija 'una visión común' donde las partes tienen su 'hoja de ruta'. ${ }^{33}$ Los puntos ' $\mathrm{f}$ ' (progresividad), ${ }^{34}$ ' $\mathrm{g}$ ' (no regresividad $)^{35} \mathrm{e}$ 'i' materializan ciertos mínimos como un ideal, sin obstaculizar que mejores garantías sean consagradas por los Estados individualmente (v. punto 'i'). Ahora bien, la finalidad de la Decisión de Santiago es compeler las partes del instrumento a actuar en armonía con el principio 10. Los Estados reunidos en Guadalajara construyeron la llamada Hoja de Ruta y acordaron implementar dicho principio, consecuencia del informe de la primera reunión donde acordaron los puntos focales de la discusión de la DP10. ${ }^{36}$

Ciertamente, las últimas páginas de la Decisión de Santiago tienen en cuenta el plan de Guadalajara, para "dar inicio a la negociación del instrumento regional sobre acceso a la información, la participación y la justicia en asuntos ambientales [...]" ${ }^{37}$ Dicha decisión acompañada de varios dispositivos, ${ }^{38}$ como grupos de trabajo, comisión de negociación,

antecedente para la negociación. Pigrau, Antoni (coord.), Acceso a la información, participación pública y acceso a la justicia: diez años del Convenio de Aarbus, Atelier, Barcelona, 2008.

32 Organización de las Naciones Unidas, Declaración sobre el Principio 10..., op. cit.

33 Comisión Económica para América Latina y el Caribe, Visión de Lima..., op. cit., p. 1.

34 “f) Progresividad. Se debe avanzar de forma progresiva hacia la cabal aplicación del principio 10 y evitar cualquier retroceso, reconociendo las circunstancias de cada país respecto de los derechos de acceso". Ibid., p. 3.

35 "g) No regresividad. El instrumento debe construirse sobre la base de los acuerdos ya alcanzados en la región y aspirar al fortalecimiento de las legislaciones nacionales o de los acuerdos internacionales para la cabal aplicación de los derechos de acceso, en coherencia con el objetivo de la Declaración". Ibid.

36 Comisión Económica para América Latina y el Caribe, Informe de la primera reunión de puntos focales designados por los gobiernos de los países signatarios de la Declaración sobre la Aplicación del Principio 10 de la Declaración de Río sobre el Medio Ambiente y el Desarrollo en América Latina y el Caribe, Nu. Cepal Doc. LC/L.3565, 6 y 7 de noviembre de 2012.

37 Comisión Económica para América Latina y el Caribe, Decisión de Santiago..., op. cit.

38 Calderón Valencia, Felipe, "Le contrôle a posteriori de la constitutionnalité des lois en droit français et colombien, éléments de compréhension d'une culture constitutionnelle”, 
modalidades de participación del público y mesa directiva. Su finalidad es darle forma normativa a las voluntades estatales, haciendo posible que ARP10 propulse los ideales de Río 92 y Río+20 e imponer obligaciones positivas y de gestión.

En consecuencia, se plantearon dos espacios de participación como parte de los compromisos para concretar el ARP10 desde la transparencia y la participación; uno del orden internacional y otro del orden interno. El orden internacional posee tres niveles de participación: ${ }^{39}$ presencial, informativo y participativo. Estos se apoyan en la representación así: 6 representantes del público latinoamericano (2 principales y 4 alternos) hacen el puente entre las propuestas del público y la mesa directiva de la negociación. ${ }^{40}$

Veamos detalladamente los tres niveles. Primero: el nivel presencial permite al público estar presente en las reuniones, física y virtualmente, y se puede intervenir sin derecho al voto. Segundo: en el nivel informativo, el público puede a acceder a documentación oficial; comparte y obtiene información con otros participantes; presenta propuestas escritas a los delegados de los gobiernos, expertos y organismos internacionales. ${ }^{41}$ Tercero: el nivel participativo permite al público pedir la palabra al presidente durante las sesiones de la negociación y las sesiones de la mesa directiva. Se otorga la palabra según el orden de las solicitudes.

Finalmente, en Santiago de Chile (5 al 7 de mayo de 2015) tuvo lugar la primera reunión del comité de negociación con participación del público. Las partes acordaron realizar consultas internas y preparar su intervención en las negociaciones, porque el espíritu del ARP10 busca favorecer la participación del público en cada país con mecanismos conformes a la legislación y prácticas internas. ${ }^{42}$

Université Pantéon-Assas (París II), en https://www.theses.fr/194933792, p.15, consulta del 10 de diciembre de 2017.

39 Comisión Económica para América Latina y el Caribe, Plan de acción hasta 2014 ..., op. cit., pp. 12-13.

40 Comisión Económica para América Latina y el Caribe, Decisión de Santiago..., op. cit.

41 Existe un mecanismo público en la web de la Cepal donde los interesados pueden suscribirse para obtener información de los avances del acuerdo. Comisión Económica para América Latina y el Caribe, "Mecanismo público regional", en http://www.cepal. org/es/registro-Principio10, consulta del 28 de febrero de 2017.

42 Comisión Económica para América Latina y el Caribe, Informe de la primera reunión del comité de negociación del Acuerdo Regional..., op. cit., p. 24. 
En síntesis, las obligaciones establecidas en la Decisión de Santiago son más concretas y pretenden que se construya un mecanismo regional que hará efectivo el principio 10 (e.g., los derechos de acceso son moldeados desde la logística misma de los puntos 9 a 13). ${ }^{43}$ En este procedimiento, Estados y público elaboran un documento previo entregando insumos a la Cepal. Lo más innovador en este trámite es que el público puede proponer las modificaciones que considere necesarias.

Se percibe que el siglo XXI trae consigo nuevas formas de abordar las necesidades y los riesgos identificados en Río 92. Así, luego de ocho negociaciones del ARP10, puede decirse que instrumentos como El Futuro que Queremos y la Decisión de Santiago sentaron las bases para lograr algo tan difícil como discutir un tratado regional 'a puertas abiertas'. ${ }^{4}$

\section{Negociación del Acuerdo Regional sobre el Principio 10: la oportunidad de pasar a la acción}

Tres temas resumen los desafíos de la negociación del ARP10: (2.1) aprobar estándares de protección robustos y ambiciosos; (2.2) evitar la remisión del articulado a las legislaciones internas; y (2.3) alcanzar un acuerdo vinculante. De no asumirse estos desafíos de conformidad con los compromisos adquiridos por las partes en la etapa de planeación, podrían convertirse en una amenaza para concretar las garantías del principio 10.

\subsection{Aprobar estándares de protección robustos y ambiciosos}

De acuerdo con la Declaración sobre el Principio 10, la Hoja de Ruta, la Visión de Lima y la Decisión de Santiago, se aspira alcanzar una visión común que defienda la importancia y los beneficios de los derechos de acceso para su cabal implementación en la región. ${ }^{45}$ Aquí, se resalta el papel de la Cepal, que, como secretaría técnica de la negociación, propuso un articulado preliminar fundamentado en los diferentes ordenamientos

43 Comisión Económica para América Latina y el Caribe, Decisión de Santiago..., op. cit., p. 5.

44 Naciones Unidas Uruguay, "Documento de posicionamiento. Proceso preparatorio nacional hacia Río+20 de la sociedad civil 2012", p. 28, en https://sustainabledevelopment. un.org/content/documents/811MVOTMA_-_Documento_hacia_Rio_20.pdf, consulta del 24 de febrero de 2017.

45 Comisión Económica para América Latina y el Caribe, Visión de Lima..., op. cit. 
jurídicos de la región ${ }^{46}$ y se ha encargado de recordar a las partes los compromisos internacionales básicos por respetar. ${ }^{47}$

No obstante, el propósito de una visión común se debilita como consecuencia de las distintas propuestas de reforma del articulado preliminar. En efecto, la observación directa y participativa de las negociaciones del ARP10 muestra que una constante ha sido hacer más laxos los deberes de los Estados. El público ha exigido trascender el limitado enfoque de los marcos jurídicos nacionales para garantizar la eficacia del principio de progresividad en la región. ${ }^{48}$ Llama, entonces, la atención la sustitución de disposiciones que contenían deberes estatales expresos y directos por otros enunciados donde las partes no asumen una 'mayor obligación'. Se aprecia en los textos compilados de la negociación del ARP10 las reiteradas solicitudes de cambiar el deber de "garantizar" por el de "promover", "fomentar" o "facilitar". 49 Algunas delegaciones insisten en reemplazar verbos que impliquen el cumplimiento de obligaciones directas de las autoridades en cada país, pese a que son claros los deberes establecidos

46 Ordenado así: primero: antecedentes que compilan los acuerdos para la negociación y fuentes del articulado preliminar (diagnóstico de las legislaciones internas, aportes de los Estados, insumos del grupo de expertos, contribuciones del público). Segundo: preámbulo reiterando compromisos y estándares internacionales. Tercero: los artículos $\left(1^{\circ}-10\right)$ que consagran objetivo, definiciones, principios, aplicación, obligaciones generales, estándares. Cuarto: los artículos (11-25) sobre recursos, órganos de administración, implementación, seguimiento, evaluación, solución de controversias, condiciones de entrada en vigor. Comisión Económica para América Latina y el Caribe, Documento preliminar del instrumento regional sobre el acceso a la información, la participación pública y el acceso a la justicia en asuntos ambientales en América Latina y el Caribe, Nu. Cepal Doc. LC/L.3987, 5 de mayo de 2015.

47 Publica compilaciones de los estándares internacionales y regionales para consulta en la negociación. Organización de las Naciones Unidas \& la Comisión Económica para América Latina y el Caribe, Sociedad, derechos y medio ambiente: estándares internacionales de derechos humanos aplicables al acceso a la información, a la participación pública y al acceso a la justicia, LC/W.712, noviembre de 2016; y Acceso a la información, participación y justicia en temas ambientales en América Latina y el Caribe: situación actual, perspectivas y ejemplos de buenas prácticas, LC/L.3549/Rev2, octubre de 2013.

48 Representantes electos del público del principio 10, "Comunicado de prensa. El llamado a una mayor voluntad política”, Santiago de Chile, 27 de noviembre de 2017, en http:// www.dar.org.pe/archivos/docs/p10/np_d1.pdf, consulta del 28 de noviembre de 2017. 49 Textos de las reuniones: ver Comisión Económica para América Latina y el Caribe, "Reunión del comité de negociación. Principio 10 en América Latina y el Caribe", en https:/ / www.cepal.org/es/organos-subsidiarios/reunion-comite-negociacion-principio-10-america-latina-caribe, consulta del 10 de diciembre de 2017. 
en diferentes tratados de derechos humanos adoptados por los Estados partes. ${ }^{50}$ Esto, en palabras del público, "socava el espíritu ambicioso que se supone debería dirigir esta negociación" ${ }^{51}$ y debilita las obligaciones de satisfacción de los derechos de acceso.

Como ejemplo de esta tendencia pueden citarse los cambios que ha tenido el articulado preliminar en los asuntos de: acceso a la información ambiental gratuita, disponibilidad de la información en custodia de las autoridades (art. $6^{\circ}$ ) y acceso a la justicia ambiental (art. $9^{\circ}$ ).

En materia de acceso a la información ambiental, el texto preliminar del ARP10 contenía el deber expreso de garantizar la gratuidad en la entrega de información (art. $6^{\circ}$, num. 18) y la disponibilidad de la información en custodia de la autoridad — cualquiera sea su formato- (art. $6^{\circ}$, num. 1). ${ }^{52}$ En las dos disposiciones se propuso reemplazar el deber de "garantizar" por fórmulas como "promover" el acceso a información gratuita ${ }^{53}$ y "promover" acciones según la legislación interna para que "sea pública y se le dé la mayor publicidad". ${ }^{44}$ Igual sucedió con la propuesta de eliminar el deber de garantizar el acceso a la justicia en asuntos ambientales (art. $\left.9^{\circ}\right)$ por la obligación más laxa de "facilitar" tal acceso. ${ }^{55}$

50 Organización de las Naciones Unidas y Comisión Económica para América Latina y el Caribe, Sociedad, derechos y medio ambiente..., op. cit., pp. 19-382; Programa de las Naciones Unidas para el Medio Ambiente, Directrices para la elaboración de legislación nacional sobre el acceso a la información, la participación del público y el acceso a la justicia en asuntos ambientales, Decisión SS.XI/ 5 parte A, 26 de febrero de 2010.

51 Cuevas, Euren, "Palabras motivadoras para los delegados por Euren Cuevas Medina, director ejecutivo de Isaproma (República Dominicana)", Santiago de Chile, 23 de noviembre de 2016, p. 2, en http://media.wix.com/ugd/0cd7e7_7b6bb081a42a4ff8a909d015d650f951. pdf, consulta del 23 de febrero de 2017.

52 Comisión Económica para América Latina y el Caribe, Documento preliminar..., op. cit., pp. 12-15.

53 Comisión Económica para América Latina y el Caribe, Texto compilado por la mesa directiva que incluye las propuestas de texto de los paises relativas al preámbulo y los artículos $1^{\circ}$ a 10 del documento preliminar del Acuerdo Regional sobre el Acceso a la Información, la Participación Pública y el Acceso a la Justicia en Asuntos Ambientales en América Latina y el Caribe. Cuarta versión, NU. Cepal Doc. LC/L.4059/Rev.3, 13 de septiembre de 2016, p. 25.

54 Propuesta de la delegación mexicana. Ibid., p. 20.

55 Propuesta de las delegaciones de Colombia y Jamaica. Comisión Económica para América Latina y el Caribe, Texto compilado por la mesa directiva que incluye las propuestas de texto de los países relativas al preámbulo y los articulos $1^{\circ}$ a 10 del documento preliminar del Acuerdo Regional sobre el Acceso a la Información, la Participación Pública y el Acceso a la Justicia en Asuntos 
Estas propuestas fueron acogidas, en parte, en la séptima reunión (31 de julio al 4 de agosto de 2017, Buenos Aires), en la que se convino el artículo $6^{\circ}$ y se avanzó en el examen del artículo $9^{\circ} .{ }^{56} \mathrm{Si}$ bien la última versión del ARP10 establece el deber de cada parte de "garantizar" el derecho de acceso a la información ambiental que está bajo su custodia, limita su entrega "sin costo" siempre que no se requiera reproducción o envío; se aprobó una fórmula intermedia. En todo caso, la regulación anticipada de costos 'razonables' y las posibles excepciones de su pago a personas en condición de vulnerabilidad se remite a la legislación interna. ${ }^{57}$

En el caso del artículo $9^{\circ}$ sobre acceso a la justicia, la séptima reunión no pudo llegar a un acuerdo sobre los verbos que deben fundamentar el deber de los Estados partes. ${ }^{58}$ Es por ello que el texto se retomó en la octava reunión. Luego de tres versiones, y de intervenciones de los expertos invitados y el público, se convino un lenguaje más exigente: "Cada parte garantizará el derecho de acceder a la justicia en asuntos ambientales de acuerdo con las garantías del debido proceso". ${ }^{5}$

Como respuesta a todo lo anterior, en la octava reunión, los representantes del público presentaron una comunicación firmada por 63 organizaciones ambientales de la región, en la que exigen a los Estados partes considerar los compromisos asumidos antes de la negociación y los estándares del Sistema Interamericano. ${ }^{60}$ Cabe citar, igualmente, que en estos casos se desconoce la jurisprudencia reiterada de la Corte Interamericana

Ambientales en América Latina y el Caribe. Quinta versión, NU. Cepal Doc. LC/L.4059/Rev.4, 26 diciembre de 2016, p. 32.

56 Comisión Económica para América Latina y el Caribe, Texto compilado por la mesa directiva que incluye las propuestas de texto de los países relativas al preámbulo y los articulos $1^{\circ}$ a 10 del documento preliminar del Acuerdo Regional sobre el Acceso a la Información, la Participación Pública y el Acceso a la Justicia en Asuntos Ambientales en América Latina y el Caribe. Séptima versión, NU. Cepal Doc. LC/L.4059/Rev.6, 6 de septiembre de 2017, p. 3.

57 Ibid., pp. 20-21.

58 Ibid., p. 27.

59 Comisión Económica para América Latina y el Caribe, "Octava reunión del comité de negociación del Acuerdo Regional sobre el Acceso a la Información, la Participación Pública y el Acceso a la Justicia en América Latina y el Caribe (principio 10)", en https:/ / negociacionp10.cepal.org/8/es, consulta del 10 de diciembre de 2017.

60 Representantes electos del público del principio 10, "Un acuerdo sobre el principio 10 vinculante que no debilite estándares en acceso a la información, participación y justicia ambiental de la región", 29 de noviembre de 2017, en http://www.dar.org.pe/noticias/ pronunciamiento_p10nov17/n, consulta del 10 de diciembre de 2017. 
de Derechos Humanos, que fija la obligación de garantía en un conjunto de medidas concretas para eliminar cualquier obstáculo al pleno disfrute de los derechos. Este tribunal dejó claro que las obligaciones de los Estados —en acceso a la información y acceso a la justicia— sobrepasan la promoción del disfrute de los derechos. ${ }^{61}$

Infortunadamente, debilitar las garantías de los derechos de acceso es una tendencia en la negociación del ARP10 y un desfavorable mensaje político a la comunidad internacional, porque espera que el resultado sea un "modelo" de protección y promoción de la democracia ambiental "para que se tomen pasos similares en otras regiones y países". ${ }^{62}$

\subsection{Evitar la remisión del articulado a las legislaciones internas}

La falta de un estándar internacional de garantías mínimas de protección a los derechos de acceso produce que la remisión a la regulación interna sea un criterio de modificación del ARP10. No obstante, este argumento resulta incongruente si se cuentan los tratados de derechos humanos ratificados por las partes, las observaciones y las recomendaciones de los organismos de derechos humanos.

Por tal razón, un reto de la negociación ha sido crear un instrumento regional que concilie las especificidades de cada Estado en la tensión

61 Medidas concretadas en deberes como: prevención que incluye medidas de carácter jurídico, administrativo y político para salvaguarda de los derechos humanos; protección de personas frente amenazas de agentes privados o públicos; investigación efectiva y sanción de violaciones de derechos humanos; reparación de víctimas; y cooperación con organismos internacionales. Ferrer, Eduardo \& Pelayo, Carlos, “La obligación de 'respetar' y 'garantizar' los derechos humanos a la luz de la jurisprudencia de la Corte Interamericana", Estudios Constitucionales, 2009, 10, (2), pp. 155-162. Compilación de estándares de acceso a la justicia del Sistema Interamericano en: Comisión Interamericana de Derechos humanos, El acceso a la justicia como garantía de los derechos económicos, sociales y culturales: estudio de los estándares fijados por el sistema, OEA/Ser.L/V/II.129, Doc. 4, 7 de septiembre de 2007. En materia de acceso a la información: Comité de Derechos Humanos, Observación General No 34, párrs. 18 y 19; Comité de Derechos Humanos, Observación General No 34, artículo 19: Libertad de opinión y libertad de expresión (CCPR/C/GC/34), párr. 18, 2011.

62 "Declaración de los expertos de las Naciones Unidas sobre la negociación de la Cepal de un instrumento regional sobre democracia ambiental", 22 de octubre de 2015, en http:/ / www.ohchr.org/SP/NewsEvents/Pages/DisplayNews.aspx?NewsID=16630\&LangID=S, consulta del 25 de febrero de 2017. 
entre: establecer dichos mínimos sin desincentivar a los ordenamientos jurídicos aventajados. Zanjar esta dificultad hace reflexionar sobre si las partes asumen el futuro del ARP10 como la oportunidad de fortalecer las capacidades internas.

Expresiones como "de conformidad con las legislaciones internas", "en consonancia con la legislación nacional" o "en el marco de la legislación nacional” afecta los siguientes asuntos: definición de personas en situación de vulnerabilidad; ${ }^{3}$ protección y apoyo a los defensores del medio ambiente; ${ }^{64}$ promoción de los derechos de acceso en igualdad de condiciones; ${ }^{65}$ disponibilidad y publicidad de la información pública; ${ }^{66} \mathrm{de}$ negación total o parcial del acceso a la información; ${ }^{67}$ plazos de respuesta a solicitudes de información; ${ }^{68}$ y garantía de acceso a órganos judiciales o autónomos para impugnar decisiones relacionadas con asuntos ambientales. ${ }^{69}$

Es imposible afirmar que entre los asuntos citados existen unos más importantes que otros. No obstante, por cuestiones metodológicas y por la preocupante situación de los defensores ambientales, ${ }^{70}$ vale la pena ampliar el análisis de su protección con el estudio de dos disposiciones.

63 Artículo $2^{\circ}$. Definiciones. Comisión Económica para América Latina y el Caribe, Texto compilado por la mesa directiva que incluye las propuestas de texto de los países relativas al preámbulo y los artículos $1^{\circ}$ a 10 del documento preliminar del Acuerdo Regional..., op. cit., p. 12.

64 Artículo $5^{\circ}$, ibid., p. 18.

65 Delegación mexicana, redacción del artículo $5^{\circ}$, numeral 11. Ibid., p. 43.

66 Ibid. Delegaciones mexicana y jamaiquina, redacción del artículo $6^{\circ}$, numeral 1 . Aunque los delegados de Uruguay y Paraguay solicitaron eliminar la remisión a la legislación interna en: promoción de facilitar condiciones básicas para solicitud de información ambiental (art. $6^{\circ}$, num. 2).

67 Artículo 60, numeral 5. Ibid., p. 22.

68 Artículo 6 ${ }^{\circ}$, numeral 12. Ibid., p. 24.

69 Artículo $9^{\circ}$, numeral 2. Ibid., p. 33.

70 Además, abordar este tema se justifica bajo el concepto de corporate social responsibility (CSR). La doctrina reciente de la Corte Suprema de Justicia de Estados Unidos ha logrado avanzar en la protección transnacional de los derechos humanos y de los defensores en virtud del Alien Tort Statute (ATS). Esta norma atípica promulgada en 1789 ha ido construyendo otro concepto útil a esta lucha: corporate social liability. Calderón Valencia, Felipe, Valencia DeLara, Pilar \& Escobar Sierra, Manuela, "Current status of the discussion on human resources management: an alternative view from corporate social responsibility and law", en Da Silva Pereira, P. A., Titrek, O. \& Sezen-Gultekin, G. (eds.), ICLEL 17 Conference Proceeding Book, 3rd International Conference on Lifelong Education and Leadership for All, ICLEL Conferences, Sakarya, 2017, p. 754. 
En primer lugar, el artículo 5.5 (obligaciones generales) del documento preliminar establece el deber de reconocimiento, protección y apoyo a las “asociaciones, organizaciones, grupos o individuos" que defienden el medio ambiente. ${ }^{71}$ Se propusieron modificaciones para incluir la obligación expresa de los Estados de velar para que los defensores no sean "acosados", "perseguidos", "intimidados" o "sometidos a medidas coactivas ilegítimas"; sin embargo, están limitadas al alcance de las legislaciones internas y, por lo tanto, a la falta de consenso sobre medidas concretas de protección. ${ }^{72}$

Las normas internas pueden ser derogadas o modificadas en cualquier momento y el hecho de contar con un instrumento internacional fuerte es una forma de asegurar los estándares ya fijados. ${ }^{73}$ Sobre la remisión del articulado a los ordenamientos internos, se comparten las siguientes inquietudes de los representantes del público: ¿qué sucede cuando las legislaciones de los Estados son diferentes? y ¿qué ocurre cuando no hay legislación al respecto?

En segundo lugar, el artículo $9^{\circ}$ bis es la norma que garantiza la protección a los defensores de derechos humanos en asuntos ambientales, según lo discutido en la séptima reunión de la negociación ${ }^{74}$ y lo

71 Comisión Económica para América Latina y el Caribe, Texto compilado por la mesa directiva que incluye las propuestas de texto de los paises relativas al preámbulo y los artículos $1^{\circ}$ a 10 del documento preliminar del Acuerdo Regional..., op. cit., p. 11. "El término defensores de los derechos humanos ambientales hace referencia a las personas y los grupos que, a título personal o profesional y de forma pacífica, se esfuerzan por proteger y promover los derechos humanos relacionados con el medio ambiente, en particular el agua, el aire, la tierra, la flora y la fauna". Organización de las Naciones Unidas, Situación de los defensores de los derechos humanos. Informe del relator especial sobre la situación de los defensores de los derechos bumanos, Sr. Michel Forst, A/71/281, 3 de agosto de 2016, p. 6.

72 Artículo $5^{\circ}$. Comisión Económica para América Latina y el Caribe, Texto compilado por la mesa directiva que incluye las propuestas de texto de los paises relativas al preámbulo y los articulos $1^{\circ}$ a 10 del documento preliminar del Acuerdo Regional..., op. cit., p. 18.

73 Representantes electos del público del principio 10, "Informe de las y los representantes electos del público acerca de la tercera reunión del comité de negociación del Acuerdo Regional sobre el Acceso a la Información, la Participación Pública y el Acceso a la Justicia en Asuntos Ambientales en América Latina y el Caribe", 13 de abril de 2016, en http:// media.wix.com/ugd/0cd7e7_c66cd76f242c4a589b284f1ae1d85157.pdf, consulta del 25 de febrero de 2017.

74 Texto preparado por Costa Rica, Panamá, Paraguay, Perú y Chile. 
convenido en la octava, el 30 de noviembre de 2017. El texto finalmente acordado es el siguiente:

1. Cada parte garantizará un entorno seguro y propicio en el que las personas, grupos y organizaciones que promuevan y defienden derechos humanos en asuntos ambientales puedan actuar sin amenazas, restricciones e inseguridad.

2. Cada parte tomará las medidas adecuadas y efectivas para reconocer, proteger y promover todos los derechos de los defensores de los derechos humanos en asuntos ambientales, incluidos su derecho a la vida, integridad personal, libertad de opinión y expresión, derecho de reunión y asociación pacíficas y derecho de circular libremente, [a]sí como su capacidad para ejercer sus derechos de acceso.

3. Cada parte tomará medidas apropiadas, efectivas y oportunas para prevenir, investigar y sancionar ataques, amenazas o intimidaciones que los defensores de los derechos humanos en asuntos ambientales puedan sufrir en el ejercicio de los derechos contenidos en el presente acuerdo.

Por el contrario, el texto preparado para la octava reunión tenía cuatro numerales dedicados, en su orden, a: definir el sujeto de protección; proteger sus derechos; asignar deberes estatales; y crear mecanismos para la no repetición (e.g., 'grupo de trabajo ad hoc'). Durante la negociación, las delegaciones lo redujeron a tres numerales, reubicando el primero en las definiciones $\left(\operatorname{art} .2^{\circ}\right.$ ) y el cuarto entre las tareas de la primera reunión de la conferencia de las partes (art. 12.4.b).

Los temas álgidos de la negociación del artículo $9^{\circ}$ bis fueron tres. Primero, agregar los derechos a la vida y la integridad personal al segundo numeral; la dificultad radicaba en poner de acuerdo al público con las delegaciones sobre si era mejor consagrar ciertos derechos o simplemente consignar el término "todos los derechos". Salomónicamente, se dejaron ambas opciones, evitando así la simple remisión a los ordenamientos internos. Segundo, saber si los defensores eran 'defensores de derechos humanos' o si eran concretamente 'en asuntos ambientales'. Tercero, determinar si la protección a los defensores debía estar ligada a la capacidad de los Estados. En efecto, el numeral 3 estuvo a punto de consagrar una protección supeditada a capacidades y procedimientos estatales propios, alejándose de la eficacia del principio de progresividad con la precitada remisión. 
La negociación del artículo $9^{\circ}$ bis demostró que es posible llegar a un consenso sobre un estándar mínimo evitando la recurrente remisión a las legislaciones internas. Sin duda, este resultado se alcanzó gracias a las propuestas e intervenciones del público que permitieron comprender que la actual situación de los defensores requiere de un estándar robusto que oriente los ordenamientos internos, porque estos no han demostrado ser una barrera eficaz contra la persecución y el asesinato. La intensificación de la criminalización, desaparición forzada, tortura y homicidio de los defensores requería un verdadero compromiso de las partes para establecer unas reglas comunes de protección. ${ }^{75}$

No hay que olvidar que en 2016 Michel Forst ${ }^{76}$ insistió en que la protección de los defensores ambientales es esencial para la protección general del ambiente. Denunció que la impunidad es común en los asesinatos de defensores, pues existe una "deplorable [...] ineficacia de las medidas de protección $[\ldots ..]{ }^{77}$ Señaló, además, que "en casi todos los países afectados de América Latina, el gobierno y los agentes empresariales están involucrados". 78

Es por ello que el relator instó a los Estados a construir un entorno propicio para la defensa de los derechos que implica revisar permanentemente: leyes, reglamentos, políticas y medidas coercitivas que garanticen que las empresas respeten los derechos de los defensores. Se resalta la exhortación a las partes del ARP10 para acelerar su conclusión, teniendo en cuenta el carácter urgente que representa el entorno hostil de la región. ${ }^{79}$

75 Según el informe de Front Line Defenders, “en 2016, la defensa de los derechos humanos en América siguió siendo una labor extremadamente peligrosa”. De 143 asesinatos reportados, los defensores de los derechos ambientales fueron los más afectados. Front Line Defenders, "Informe anual sobre los/as defensores/as de derechos humanos en riesgo 2016", en https://www.frontlinedefenders.org/es/resource-publication/ annual-report-human-rights-defenders-risk-2016, consulta del 25 de febrero de 2017. La organización Global Witness reportó el 2015 como el peor de la historia. Esta organización publicó las estadísticas de homicidios de defensores entre 2010 y 2015 en Latinoamérica. Global Witness, "En terreno peligroso. Reporte”, 20 de junio de 2016, en https://www. globalwitness.org/en/reports/terreno-peligroso/, consulta del 25 de febrero de 2017.

76 Relator especial sobre la situación de los defensores de los derechos humanos.

77 Organización de las Naciones Unidas, Situación de los defensores de los derechos humanos..., op. cit., p. 12.

78 Ibid.

79 Ibid., p. 10. 
De ahí que este artículo $9^{\circ}$ bis sea de celebrar frente a las recomendaciones del relator. ${ }^{80}$

\subsection{Alcanzar un acuerdo vinculante}

Alcanzar un acuerdo obligatorio es una prueba contundente de la intención de los Estados de pasar de la etapa de planeación a una etapa de acciones concretas del principio 10. No obstante, resulta paradójico que la fuerza vinculante del instrumento de la negociación no haya sido acordada desde el principio de la negociación. Así se consigna en el informe de la cuarta reunión de los puntos focales (Santiago de Chile en 2014), en donde las partes convinieron que la naturaleza del acuerdo sería definida en el transcurso de la negociación. ${ }^{81}$ Aunque en la doctrina del derecho internacional ambiental se acepte el predominio del soft law como un rasgo característico que le permite su fluidez, ${ }^{82}$ no es menos cierto que, aunque "la falta de coerción es políticamente útil", esta "se traduce en problemas operativos para la solución de controversias". ${ }^{83}$

Sobre el tema, los expertos invitados a observar la negociación han sido enfáticos en afirmar que "sin un enfoque vinculante difícilmente se le puede dar operatividad al contenido de un instrumento que regule estas materias", 84 esto es, los derechos de acceso.

No se discute en este trabajo la conveniencia de un instrumento obligatorio que haga efectivos los estándares acordados en la negociación, pues, de ser así, cinco años de preparación y discusión del articulado fueron en vano. Consideramos, por el contrario, que la cláusula de obligatoriedad debería estar acompañada de — por lo menos- dos mecanismos que den eficacia y operatividad a los estándares acordados. Primero: la creación de un órgano competente del control y la entrega de pautas para

\footnotetext{
$80 \quad$ Ibid., pp. 32-33.

81 En esta reunión, Guatemala, Panamá, Paraguay, República Dominicana, Uruguay, Chile, Costa Rica, Perú y San Vicente y Las Granadinas manifestaron voluntad para adoptar un convenio vinculante. Comisión Económica para América Latina y el Caribe, Informe de la cuarta reunión de los puntos focales..., op. cit., pp. 17, 21.

82 Juste, José, Derecho internacional del medio ambiente, McGraw-Hill, Madrid, 1999, p. 44.

83 Guzmán, Tratados ambientales..., op. cit., p. 74.

84 Comisión Económica para América Latina y el Caribe, Conversatorio sobre la naturaleza del instrumento regional. Resumen de respuestas y comentarios de expertos en derecho público ambiental internacional, NU. Cepal Doc. LC/L.3938, $1^{\circ}$ de agosto de 2014, p. 10.
} 
el cumplimiento de los compromisos por los Estados partes. Esto puede lograrse otorgando una función de control al órgano subsidiario que establezca la Conferencia de las Partes (art. 12.5). Segundo: la inclusión de la obligación de estos últimos de presentar informes periódicos.

En los sistemas de protección de derechos humanos, los órganos creados como consecuencia de la aprobación de un tratado han establecido diferentes prácticas que les permiten verificar el grado de cumplimiento en la protección de los derechos y su promoción. ${ }^{85}$ Algunas de estas prácticas son: recibir y examinar informes periódicos, formular directrices, hacer investigaciones, emitir recomendaciones y observaciones generales. Lo anterior podría asegurar el cumplimiento de los estándares que sean finalmente acordados.

\section{Conclusión}

El derecho ambiental latinoamericano se ha nutrido principalmente del derecho internacional producido en las Cumbres de la Tierra, en la llamada primera etapa o etapa de planeación. Además, podría decirse que su identidad está construyéndose en la segunda etapa o etapa de concreción, en torno al componente democrático emanado del principio 10. Prueba de ello es el gran avance de la negociación del ARP10 y la activa participación del público en su construcción como un elemento inédito.

Tal avance no puede comprenderse sino a partir de la comprensión de la evolución histórica de las fuentes de la negociación desde Río 92 - y en general, de las Cumbres_ - y a la par de la evolución del derecho de los derechos humanos.

La negociación del ARP10 sigue siendo una oportunidad para responder a las diferentes críticas que aluden a la 'falta de dientes' de los acuerdos en materia medioambiental. Sin embargo, persiste el reto de los Estados partes de trascender una visión limitada del futuro, influenciada por la declaración Río+20, cuyo pilar fundamental es el fortalecimiento de la gobernanza internacional ambiental y los mecanismos necesarios que permitan la firma de un instrumento robusto y efectivo de garantías de los derechos de acceso que dé inicio a una etapa de acciones concretas.

85 Oficina del Alto Comisionado de las Naciones Unidas para los Derechos Humanos, El sistema de tratados de los derechos humanos de las Naciones Unidas, Naciones Unidas, Ginebra, 2012, p. 25. 


\section{Referencias}

Bastida, Ana \& Bustos, Luis, “Towards regimes for sustainable mineral resource management. Constitutional reform, law and judicial decisions in Latin America", en Carbonnier, G., Campodónico, H. \& Vázquez, S. T. (eds.), Alternative pathways to sustainable development: lessons from Latin America, Brill, Geneva, 2017, pp. 242-244.

Bryant, Raymond, "Beyond the impasse: the power of political ecology in Third World environmental research", Area, 1997, 29, (1), pp. 5-19.

Calderón Valencia, Felipe, Valencia DeLara, Pilar \& Escobar Sierra, Manuela, "Current status of the discussion on human resources management: an alternative view from corporate social responsibility and law", en Da Silva Pereira, P. A., Titrek, O. \& Sezen-Gultekin, G. (eds.), ICLEL 17 Conference Proceeding Book, 3rd International Conference on Lifelong Education and Leadership for All, ICLEL Conferences, Sakarya, 2017, pp. 747-760.

Cabrera, Jorge, "El impacto de las declaraciones de Río y Estocolmo sobre la legislación y las políticas ambientales en América Latina", Revista Interdisciplinar de Gestión Ambiental, 2004, 6, (61), pp. 25-41.

Castro Buitrago, Erika, Derecho ambiental y gobernanza. La concertación como actuación informal de la Administración, Universidad de Medellín, Medellín, 2017.

De Wet, Erika, "The international constitutional order", International and Comparative Law Quarterly, 2006, 55, (1), pp. 51-76.

Guzmán, Patricia, Tratados ambientales internacionales y negocios, Universidad Externado de Colombia, Bogotá, 2012.

Lozano, Blanca, Derecho ambiental administrativo, Dykinson, Madrid, 2007.

Ost, Francois, Naturaleza y derecho: para un debate ecológico en profundidad, Editorial Mensajero, Bilbao, 1996.

Pigrau, Antoni (coord.), Acceso a la información, participación pública y acceso a la justicia: diez años del Convenio de Aarbus, Atelier, Barcelona, 2008.

Porter, John, Howden, Mark, Smith, Pete \& Stokholm, Michaela, "Recasting economics as if the climate and global ecology really mattered", Consilience, 2017, (17), pp. 220-229.

Rosso, Margaret, "Climate change and the law", The American Journal of Comparative Law, 2010, 58, pp. 223-255.

Sands, Philipe, Peel, Jacqueline, Fabra, Adriana \& MacKenzie, Ruth, Principles of international environmental law, Cambridge University Press, Nueva York, 2012. 


\section{Documentos oficiales}

Comisión Económica para América Latina y el Caribe, Conversatorio sobre la naturaleza del instrumento regional. Resumen de respuestas y comentarios de expertos en derecho público ambiental internacional, NU. Cepal Doc. LC/L.3938, $1^{\circ}$ de agosto de 2014.

Comisión Económica para América Latina y el Caribe, Decisión de Santiago. Cuarta reunión de los puntos focales designados por los gobiernos de los paises signatarios de la Declaración sobre la Aplicación del Principio 10 de la Declaración de Río sobre el Medio Ambiente y el Desarrollo en América Latina y el Caribe, Nu. Cepal Doc., 7 de noviembre de 2014.

Comisión Económica para América Latina y el Caribe, Documento preliminar del instrumento regional sobre el acceso a la información, la participación pública y el acceso a la justicia en asuntos ambientales en América Latina y el Caribe, Nu. Cepal Doc. LC/L.3987, 5 de mayo de 2015.

Comisión Económica para América Latina y el Caribe, Informe de la primera reunión de puntos focales designados por los gobiernos de los paises signatarios de la Declaración sobre la Aplicación del Principio 10 de la Declaración de Río sobre el Medio Ambiente y el Desarrollo en América Latina y el Caribe, Nu. Cepal Doc. LC/L.3565, 6 y 7 de noviembre de 2012.

Comisión Económica para América Latina y el Caribe, Informe de la cuarta reunión de los puntos focales designados por los gobiernos de los paises signatarios de la Declaración sobre la Aplicación del Principio 10 de la Declaración de Rio sobre el Medio Ambiente y el Desarrollo en América Latina y el Caribe, Nu. Cepal Doc. LC/L.3970, 12 de marzo de 2015.

Comisión Económica para América Latina y el Caribe, Informe de la primera reunión del comité de negociación del Acuerdo Regional sobre el Acceso a la Información, la Participación Pública y el Acceso a la Justicia en Asuntos Ambientales en América Latina y el Caribe, NU. Cepal Doc. LC/L.4046, 10 de agosto de 2015.

Comisión Económica para América Latina y el Caribe, Plan de acción hasta 2014 para la implementación de la Declaración sobre la Aplicación del Principio 10 de la Declaración de Río sobre el Medio Ambiente y el Desarrollo en América Latina y el Caribe y su hoja de ruta, NU. Cepal Doc., 17 de abril de 2013.

Comisión Económica para América Latina y el Caribe, Rio 92 y la realidad latinoamericana. Una mirada a la crisis ambiental desde el sur, NU. Cepal Doc. LC/R.1140, 4 de mayo de 2002. 
Comisión Económica para América Latina y el Caribe, Texto compilado por la mesa directiva que incluye las propuestas de texto de los paises relativas al preámbulo y los artículos $1^{\circ}$ a 10 del documento preliminar del Acuerdo Regional sobre el Acceso a la Información, la Participación Pública y el Acceso a la Justicia en Asuntos Ambientales en América Latina y el Caribe. Cuarta versión, NU. Cepal Doc. LC/L.4059/Rev.3, 13 de septiembre de 2016.

Comisión Económica para América Latina y el Caribe, Texto compilado por la mesa directiva que incluye las propuestas de texto de los países relativas al preámbulo y los artículos $1^{\circ}$ a 10 del documento preliminar del Acuerdo Regional sobre el Acceso a la Información, la Participación Pública y el Acceso a la Justicia en Asuntos Ambientales en América Latina y el Caribe. Quinta versión, Nu. Cepal Doc. LC/L.4059/Rev.4, 26 de diciembre de 2016.

Comisión Económica para América Latina y el Caribe, Texto compilado por la mesa directiva que incluye las propuestas de texto de los países relativas al preámbulo y los artículos $1^{\circ}$ a 10 del documento preliminar del Acuerdo Regional sobre el Acceso a la Información, la Participación Pública y el Acceso a la Justicia en Asuntos Ambientales en América Latina y el Caribe. Séptima versión, NU. Cepal Doc. LC/L.4059/Rev.6, 6 de septiembre de 2017 , p. 4.

Comisión Económica para América Latina y el Caribe, Visión de Lima para un instrumento regional sobre los derechos de acceso en materia ambiental, NU. Cepal Doc. LC/L.3780, 21 de febrero de 2014.

Comisión Interamericana de Derechos Humanos, El acceso a la justicia como garantía de los derechos económicos, sociales y culturales: estudio de los estándares fijados por el sistema, OEA/Ser.L/V/II.129, Doc. 4, 7 de septiembre de 2007.

Comité de Derechos Humanos, Observación General No34, artículo 19: Libertad de opinión y libertad de expresión, CCPR/C/GC/34, 2011.

Oficina del Alto Comisionado de las Naciones Unidas para los Derechos Humanos, El sistema de tratados de los derechos humanos de las Naciones Unidas, Naciones Unidas, Ginebra, 2012.

Organización de las Naciones Unidas, Declaración sobre el Principio 10: acceso a la información, participación y justicia en asuntos ambientales en América Latina y el Caribe, Nu. Doc., A/CONF.216/13, 25 de julio de 2012. Organización de las Naciones Unidas, El futuro que queremos, NU. Doc. A/ CONF.216/L.1, 19 de junio de 2012.

Organización de las Naciones Unidas, Plan de aplicación de las decisiones de la Cumbre Mundial sobre el Desarrollo Sostenible Johannesburgo (Sudáfrica), 
26 de agosto al 4 de septiembre de 2002, NU. Doc. A/CONF.199/L.3/ Add.1-13, 4 de septiembre de 2002.

Organización de las Naciones Unidas, Situación de los defensores de los derechos bumanos. Informe del relator especial sobre la situación de los defensores de los derechos humanos, Sr. Michel Forst, A/71/281, 3 de agosto de 2016.

Organización de las Naciones Unidas \& Comisión Económica para América Latina y el Caribe, Acceso a la información, participación y justicia en temas ambientales en América Latina y el Caribe: situación actual, perspectivas y ejemplos de buenas prácticas, LC/L.3549/Rev2, octubre de 2013.

Organización de las Naciones Unidas \& Comisión Económica para América Latina y el Caribe, Sociedad, derechos y medio ambiente: estándares internacionales de derechos bumanos aplicables al acceso a la información, a la participación pública y al acceso a la justicia, LC/W.712, noviembre de 2016. Programa de las Naciones Unidas para el Medio Ambiente, Directrices para la elaboración de legislación nacional sobre el acceso a la información, la participación del público y el acceso a la justicia en asuntos ambientales, Decisión SS.XI/5, parte A, 26 de febrero de 2010.

\section{Recursos de internet}

Calderón Valencia, Felipe, "Le contrôle a posteriori de la constitutionnalité des lois en droit français et colombien, éléments de compréhension d'une culture constitutionnelle", Université Pantéon-Assas (París II), p. 15, en https:/ /www.theses.fr/194933792, consulta del 10 de diciembre de 2017.

Comisión Económica para América Latina y el Caribe, "Mecanismo público regional", en http://www.cepal.org/es/registro-Principio10, consulta del 28 de febrero de 2017.

Comisión Económica para América Latina y el Caribe, “Octava reunión del comité de negociación del Acuerdo Regional sobre el Acceso a la Información, la Participación Pública y el Acceso a la Justicia en América Latina y el Caribe (principio 10)", en https://negociacionp10.cepal.org/8/es, consulta del 10 de diciembre de 2017.

Cuevas, Euren, "Palabras motivadoras para los delegados por Euren Cuevas Medina, director ejecutivo de Isaproma (República Dominicana)", 23 de noviembre de 2016, en http: //media.wix.com/ugd/0cd7 e7_7b6bb081a42a4ff8a909d015d650f951.pdf, consulta del 23 de febrero de 2017. 
Front Line Defenders, "Informe anual sobre los/as defensores/as de derechos humanos en riesgo 2016", en https://www.frontlinedefenders.org/es/resource-publication/annual-report-human-rightsdefenders-risk-2016, consulta del 25 de febrero de 2017.

Global Witness, "En terreno peligroso. Reporte", 20 de junio de 2016, en https://www.globalwitness.org/en/reports/terreno-peligroso/, consulta del 25 de febrero de 2017.

Godard, Oliver, "Le développement durable de Río de Janeiro (1992) 'a Johannesburg (2002)", CECO-803, 2003, en https:/ / halshs.archivesouvertes.fr/hal-00242971/document, consulta del 25 de febrero de 2017.

Naciones Unidas Uruguay, "Documento de posicionamiento. Proceso preparatorio nacional hacia Río +20 de la sociedad civil 2012", en https://sustainabledevelopment.un.org/content/ documents/811MVOTMA_-_Documento_hacia_Rio__20.pdf, consulta del 24 de febrero de 2017.

Organización de las Naciones Unidas, “Cumbre para la Tierra +5 , período extraordinario de sesiones de la Asamblea General para el Examen y la Evaluación de la Aplicación del Programa 21", Nueva York, 23 a 27 de junio de 1997, en http:/ /www.un.org/spanish/conferences/ cumbre\&5.htm, consulta del 25 de febrero de 2017.

Organización de las Naciones Unidas, "Declaración de los expertos de las Naciones Unidas sobre la negociación de la Cepal de un instrumento regional sobre democracia ambiental", 22 de octubre de 2015, en http://www.ohchr.org/SP/NewsEvents/Pages/DisplayNews. aspx?NewsID=16630\&LangID=S, consulta del 25 de febrero de 2017.

Organización de las Naciones Unidas, "Declaración de Río sobre el Medio Ambiente y el Desarrollo", 14 de junio de 1992, en http:/ /www. un.org/spanish/esa/sustdev/documents/declaracionrio.htm, consulta del 25 de febrero de 2017.

Organización de las Naciones Unidas, "Declaration of the United $\mathrm{Na}$ tions Conference on the Human Environment", 16 de junio de 1972, en http://www.unep.org/Documents.Multilingual/Default. asp?DocumentID=97\&ArticleID=1503\&l=en, consulta del $27 \mathrm{de}$ febrero de 2017.

Organización de las Naciones Unidas, "Programa 21", en http://www. un.org/spanish/esa/sustdev/agenda21/, consulta del 25 de febrero de 2017. 
Representantes electos del público del principio 10, "Comunicado de prensa. El llamado a una mayor voluntad política”, Santiago de Chile, 27 de noviembre de 2017, en http:/ / www.dar.org.pe/archivos/docs/ p10/np_d1.pdf, consulta del 28 de noviembre de 2017.

Representantes electos del público del principio 10, "Informe de las y los representantes electos del público acerca de la tercera reunión del comité de negociación del Acuerdo Regional sobre el Acceso a la Información, la Participación Pública y el Acceso a la Justicia en Asuntos Ambientales en América Latina y el Caribe", 13 de abril de 2016, en http:/ / media.wix.com/ugd/0cd7e7_c66cd76f242c4a589b284f1ae1d85157.pdf

Representantes electos del público del principio 10, "Un acuerdo sobre el principio 10 vinculante que no debilite estándares en acceso a la información, participación y justicia ambiental de la región”, 29 de noviembre de 2017, en http://www.dar.org.pe/noticias/pronunciamiento_p10nov17/n, consulta del 10 de diciembre de 2017. 